\title{
Channel Rectification and Signal Estimation Based on EIV Model in Massive MIMO System
}

\author{
Liang Zhong, ${ }^{1}$ Li Huang, ${ }^{2}$ and Zhengmin Kong ${ }^{3}$ \\ ${ }^{1}$ School of Mechanical Engineering and Electronic Information, China University of Geosciences, Wuhan 430072, China \\ ${ }^{2}$ College of Electronics and Information, South-Central University for Nationalities, \\ Hubei Key Laboratory of Intelligent Wireless Communications, Wuhan 430074, China \\ ${ }^{3}$ Department of Automation, Wuhan University, Wuhan 430072, China
}

Correspondence should be addressed to Li Huang; szemmah@hotmail.com

Received 18 September 2016; Revised 21 December 2016; Accepted 16 January 2017; Published 12 February 2017

Academic Editor: Raffaele Solimene

Copyright (C) 2017 Liang Zhong et al. This is an open access article distributed under the Creative Commons Attribution License, which permits unrestricted use, distribution, and reproduction in any medium, provided the original work is properly cited.

The performance of Massive MIMO is severely limited by channel estimation error, which is caused by pilot contamination and channel aging. In this paper, we propose an estimation algorithm based on the weighted total least-squares method with errors-invariables (EIV) model to alleviate the influence of pilot contamination and channel aging. Then, a channel rectification method has been investigated to diminish the inaccuracy of channel estimation. Comparing with the traditional methods, it not only helps to make the signal estimation more accurate, but also provides opportunities to correct the channel model with estimation error and update the aged channel statement information. Simulations are provided to verify the efficacy of this method.

\section{Introduction}

Massive MIMO, which is a system where base stations with a large number of antennas and tens of single-antenna user terminals (UT) deployed in each cell, has attracted attentions for its high spectrum efficiency [1]. Besides, as the number of antennas at the BS is far larger than that of UTs in each cell, the downlink channel estimation will not be feasible [2]. Therefore, time division duplex (TDD) is used and the channel is assumed to be reciprocal where the downlink channel is gained from the uplink training. During the training period, the pilots are reused at each cell as the number of orthogonal pilots is limited by coherent bandwidth and coherent time of the mobile channel [3]. So, the channel estimation is contaminated, which is called pilot contamination [4]. On the other hand, as time goes by, the channel varies, and it is different from the estimated state. This phenomenon is the so-called channel aging. The primary cause of this problem is that the antennas and those of objects/people move in the propagation medium [5]. These two impacts cause harmful influences on the uplink signal decoding and the downlink precoding, thus on the performance of the transmission.
To alleviate the effect of pilot contamination, many prior works have been proposed. For example, a spatial domain method, which uses a predetermined angular-tunable narrow beam pattern to match the desired user based on the fact that the spatial and/or temporal characters of channel coefficients of different users are different, has been proposed in [3]. The work in [6] addressed the pilot contamination in cosine modulated multitone (CMT) and took advantage of the socalled blind equalization property to mitigate the pilot contamination effect. In [7], an iterative least square projection (ILSP) method based on diagonal Jacket matrix is proposed to resolve the effects of pilot contamination as diagonal Jacket matrix has many advantages such as reducing the computational complexity and an energy harvest. Meanwhile, to avoid the channel aging, through minimizing the mean square error (MSE) in the channel prediction, channel state information (CSI) is predicted by using the aged CSI and a Wiener linear predictor to overcome channel aging effects [5]. However, less work has been done to solve the problem of channel aging and pilot contamination jointly.

Here, we consider the channel-aging-only scenario and then incorporate the effect of pilot contamination later on. The effects of channel aging and pilot contamination are 


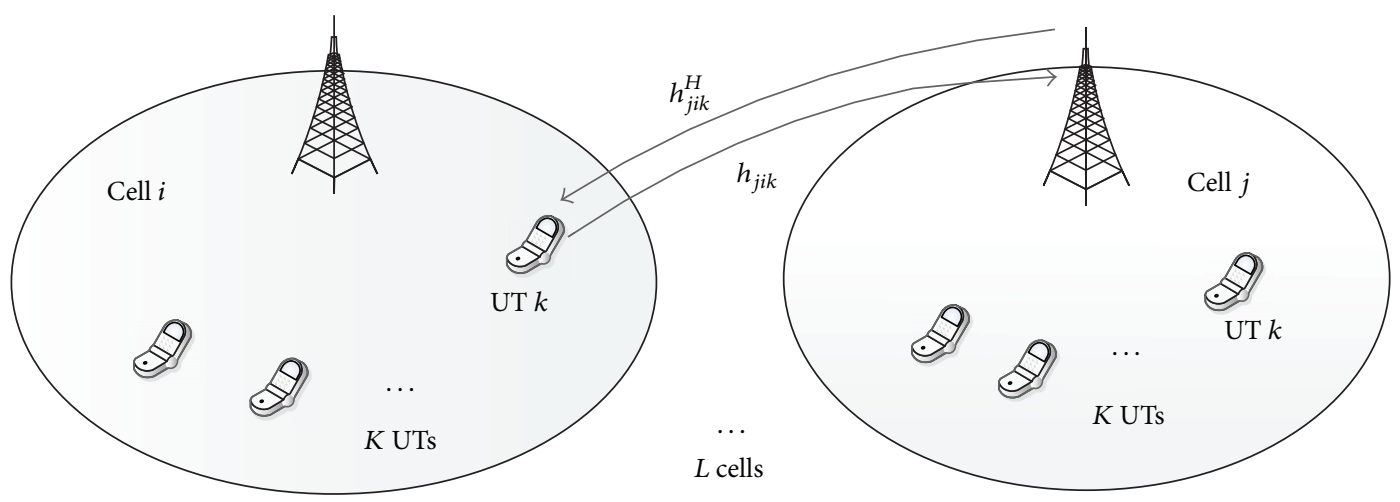

Figure 1: Massive MIMO system model under consideration.

modeled jointly, and the jointed model is then transformed into an EIV model of the heterogeneous covariance case. After that, an iterative algorithm is proposed based on weighted total least-squares (WTLS) to solve the problem, that is, to estimate the transmitted signal. With the algorithm, the channel estimate error that is caused by channel aging and pilot contamination can be derived. In the end, the CSI can be rectified according to the derived channel estimate error. Therefore, the effects of channel aging and pilot contamination, which are inherent in Massive MIMO system, can be eliminated jointly with the proposed algorithm. At the end of this paper, simulation results show that, with the rectified CSI, the signal estimation becomes more accurate with the proposed method.

Throughout this paper, the following notations are used. $\mathbf{I}_{M}$ is the unit matrix of size $M \times M \cdot(\bullet)^{-1},(\bullet)^{T},(\bullet)^{H}$ indicate the inverse, the transpose, and the conjugate transpose, respectively, and $x^{H}$ is used to denote the complex conjugate of $x$ when $x$ is a complex number. $x \sim \mathbb{C N}(a, b)$ means that $x$ satisfies the complex Gaussian distribution with mean $a$ and covariance $b$, and $\mathbf{X} \in \mathbb{C}^{M \times N}$ denotes that $\mathbf{X}$ is a matrix with size $M \times N$. Finally, $\|\bullet\|$ is the Euclidean norm. $\mathbb{E}[]$ is the expectation operation and " $\otimes$ " is the Kronecker product of two matrices.

\section{System Model}

We consider the general noncooperative multicell multiuser TDD Massive MIMO system as shown in Figure 1. This system consists of $L(L>1)$ cells. One base station and $K(K>$ 1) user terminals (UTs) are deployed in each cell. The UTs are single-antenna while the base station has $M$ antennas, where $M$ is far larger than $K$. The same frequency band is shared by the whole system. Apart from that, we assume the channel reciprocity as the propagation channel is essentially reciprocal so that the downlink channel is the Hermitian transpose of the corresponding uplink channel [2].

Here, a quasi-static channel model is assumed, so the channels remain the same within one symbol but vary from symbol to symbol. For the simplicity of analysis, we use the channel model $\mathbf{H}_{j i k}[n]=\mathbf{R}_{j i k}^{1 / 2} \mathbf{v}[n] \in \mathbb{C}^{M \times 1}$, where $n$ denotes channels at the $n$th symbol and the subscripts indicate the channel from the $k$ th UT in cell $i$ to the base station $j$ in cell. This kind of channel model, which is also known as the stationary ergodic Gauss-Markov block fading channel model, is versatile and is constituted by two parts: $\mathbf{R}_{j i k}=\mathbb{E}\left[\mathbf{H}_{j i k}[n] \mathbf{H}_{j i k}^{H}[n]\right] \in \mathbb{C}^{M \times M}$ is a deterministic correlation matrix and is independent of the symbol index $n$; $\mathbf{v}[n] \sim \mathbb{C} \mathbb{N}\left(0, \mathbf{I}_{M}\right)$ is a fast-fading channel vector $[5,8]$. Actually, $\mathbf{R}_{j i k}$ represents the general properties of the channel while $\mathbf{v}$ stands for the channels dynamic characteristics. Then, $\mathbf{H}_{j i}[n]=\left[\mathbf{H}_{j i 1}[n], \mathbf{H}_{j i 2}[n], \ldots, \mathbf{H}_{j i K}[n]\right] \in \mathbb{C}^{M \times K}$ denotes the channel from UTs in cell $i$ to the base station in cell $j$. Since the channel vectors of different users are assumed to be Gaussian random process with i.i.d. entries, the channel matrix $\mathbf{H}_{j i}[n]$ is column full-rank.

In this paper, only the uplink is considered, and it consists of the training period and the transmission period, so we analyze the received signal at the base station, such as $y_{j}$ at the base station in cell $j$ :

$$
\begin{aligned}
\mathbf{Y}_{j}[n]= & \sqrt{P_{u l}} \sum_{i=1}^{L} \mathbf{H}_{j i}[n] \mathbf{X}_{i}[n]+\mathbf{z}_{j}[n] \\
= & \sqrt{P_{u l}} \mathbf{H}_{j j}[n] \mathbf{X}_{j}[n] \\
& +\sqrt{P_{u l}} \sum_{i=1, i \neq j}^{L} \mathbf{H}_{j i}[n] \mathbf{X}_{i}[n]+\mathbf{z}_{j}[n],
\end{aligned}
$$

where $\mathbf{X}_{i}[n]=\left[x_{i 1}[n], \ldots, x_{i K}[n]\right]^{T} \sim \mathbb{C N}\left(0, \mathbf{I}_{K}\right)$ and $x_{i k}[n] \epsilon$ $\mathbb{C}^{1 \times 1}$ indicates the transmitted signal of the $k$ th UT in cell $i$ at the $n$th symbol time. $P_{u l}$ is the transmit power, $\delta_{b}^{2}$ is the noise power, and $\mathbf{z}_{j}[n] \sim \mathbb{C} \mathbb{N}\left(0, \delta_{b}^{2} \mathbf{I}_{M}\right)$ is the received white addictive Gaussian noise at base station in cell $j$.

\section{Weighted Total Least-Squares Algorithm for Signal Estimation}

The EIV observation model is conceptually symbolized as in $[9,10]$ :

$$
\mathbf{y}-e_{y}=\left(\mathbf{A}-\mathbf{E}_{\mathbf{A}}\right) \boldsymbol{\xi}
$$


where $\mathbf{y} \in \mathbb{C}^{n \times 1}, \mathbf{A} \in \mathbb{C}^{n \times m}, \boldsymbol{\xi} \in \mathbb{C}^{m \times 1}$, and $n>m=\operatorname{rank}(\mathbf{A})$. $e_{y}$ and $\mathbf{E}_{\mathbf{A}}$ are the corresponding observation error of $\mathbf{y}$ and $\mathbf{A}$, respectively, and their stochastic properties are characterized by

$$
\left[\begin{array}{l}
e_{y} \\
e_{\mathbf{A}}
\end{array}\right] \sim\left(\left[\begin{array}{l}
0 \\
0
\end{array}\right], \sigma_{0}^{2}\left[\begin{array}{cc}
\mathbf{Q}_{y} & 0 \\
0 & \mathbf{Q}_{\mathbf{A}}
\end{array}\right]\right),
$$

where $e_{\mathbf{A}}=\operatorname{vec}\left(\mathbf{E}_{\mathbf{A}}\right)$ and "vec" is the operation that converts a matrix to a column vector by stacking one column of this matrix underneath the previous one. $\mathbf{Q}_{y} \in \mathbb{C}^{n \times n}$ and $\mathbf{Q}_{\mathbf{A}} \in$ $\mathbb{C}^{n m \times n m}\left(\mathbf{Q}_{\mathbf{A}}=\mathbf{Q}_{0} \otimes \mathbf{Q}_{x}\right.$; the individual cofactor matrices $\mathbf{Q}_{0}$ have size $m \times m$ and $\mathbf{Q}_{x}$ has size $n \times n ; \mathbf{Q}_{0}$ could be singular) are the symmetric and nonnegative-definite cofactor matrices of $e_{y}$ and $e_{\mathrm{A}}$, and $\sigma_{0}^{2}$ is the variance component, which is unknown.

In the consideration Massive MIMO system, $\xi$, $\mathbf{A}$, and $y$ usually correspond to the transmitted signal, the system coefficients, and the received signal, respectively. In the following sections, we will model the signal estimation problem with the effect of pilot contamination and channel aging as EIV model.

3.1. Channel Aging. In the realistic wireless communication system, the variation of channels never stops due to the change of various factors, such as the relative movement of the transmitters and receivers, the temperature, the humidity, and the mobility of adjacent objects. Therefore, the channel changes between the training period and the transmission period of the uplink, which affects the accuracy of channel modeling and signal estimation at the base station. Here, for analysis, we adopt the channel aging model as in [5], where the channel model at $(n+1)$ th symbol is

$$
\mathbf{H}_{j i k}[n+1]=\sum_{p=0}^{P} \boldsymbol{\alpha}_{p} \mathbf{H}_{j i k}[n-p]+\mathbf{e}_{j i k}[n+1],
$$

where $\left\{\boldsymbol{\alpha}_{p}\right\}_{p=0}^{P}$ are the AR coefficients and $\mathbf{e}_{j i k}[n+1]$ is temporally uncorrelated complex white Gaussian noise process.

For simplicity, we use the first-order model to approximate the changing channel; that is,

$$
\mathbf{H}_{j i k}[n+1]=\boldsymbol{\alpha} \mathbf{H}_{j i k}[n]+\mathbf{e}_{j i k}[n+1] .
$$

It is obvious that increasing the order $P$ will improve the accuracy of channel modeling but increase the complexity as well. In this first-order model, $\alpha=J_{0}\left(2 \pi f_{d} T_{s}\right) \approx 1-\left(\pi f_{d} T_{s}\right)^{2}$, where $J_{0}(\cdot)$ is zeroth-order Bessel function of the first kind, $f_{d}$ is the maximum Doppler shift, and $T_{s}$ is the channel sampling duration; $\mathbf{e}_{j i k}[n+1] \sim \mathbb{C N}\left(0, \mathbf{R}_{j i k}-\alpha^{2} \mathbf{R}_{j i k}\right)$ is the uncorrelated channel error due to the effect of channel aging. From this model, we can easily get the idea that $\alpha$ decreases as $f_{d}$ becomes larger so that $\mathbf{H}_{j i k}[n+1]$ becomes less dependent on $\mathbf{H}_{j i k}[n]$, which coincides with our intuition that the channel changes severely if the environment has a great variation. Specially, when $f_{d} T_{s}=1 / \pi, \mathbf{H}_{j i k}[n+1]=\mathbf{e}_{j i k}[n+1]$, which means $\mathbf{H}_{j i k}[n+1]$ has nothing to do with $\mathbf{H}_{j i k}[n]$.

According to [11], compared to pilot contamination, multiuser interference and noise are negligible impacts.
Therefore, the received signal at the base station from UT $k$ in cell $j$ is

$$
\mathbf{y}_{j k}[n+1]=\sqrt{P_{u l}} \mathbf{H}_{j j k}[n+1] \mathbf{x}_{j k}[n+1]+\mathbf{z}_{j}[n+1] .
$$

By inserting (5) into (6), we can obtain the received signal when the channel aging error $\left(-\sqrt{P_{u l}} \mathbf{e}_{j j k}[n+1]\right)$ is considered, which is

$$
\begin{aligned}
\mathbf{y}_{j k} & {[n+1]-\mathbf{z}_{j}[n+1] } \\
= & \left(\alpha \sqrt{P_{u l}} \mathbf{H}_{j j k}[n]-\left(-\sqrt{P_{u l}} \mathbf{e}_{j j k}[n+1]\right)\right) \\
\cdot & \mathbf{x}_{j k}[n+1] .
\end{aligned}
$$

Assume that $\sigma_{0}^{2}=1$; then we define

$$
\begin{aligned}
& \mathbf{Q}_{y}=\delta_{0}^{2} \mathbf{I}_{M} ; \\
& \mathbf{Q}_{0}=\mathbf{I}_{M} ; \\
& \mathbf{Q}_{x}=P_{u l}\left(\mathbf{R}_{j j k}-\alpha^{2} \mathbf{R}_{j j k}\right) .
\end{aligned}
$$

Therefore, the signal estimation problem can be treated as a total least square problem, and it is modeled as an EIV model. Consequently, the estimation of $\mathbf{x}_{j k}[n+1]$ can be derived through the proposed algorithm. The performance compared to the no-channel-updating scenario is demonstrated by simulation.

3.2. Incorporating Pilot Contamination. After modeling the channel aging separately, we will derive the model of the incorporating pilot contamination, which will also be transformed into an EIV model. The uplink channel estimation at the base station is made by using the pilots or the training sequences sent by user $k, k \in[1, \ldots, K]$. Let $\tau$ be the length of the training period. $\Phi_{k}[1: \tau] \in \mathbb{C}^{\tau \times 1}$ is the pilot vector of the $k$ th user in the training period. Suppose that all cells use the same pilots set $\Phi[1: \tau]=\left[\Phi_{1}[1: \tau], \Phi_{2}[1: \tau], \ldots, \Phi_{K}[1:\right.$ $\tau]]^{T} \in \mathbb{C}^{K \times \tau}$; the pilots are normalized to meet $\boldsymbol{\Phi} \cdot \boldsymbol{\Phi}^{H}=\mathbf{I}_{K}$. So, the received signal at base station $j$ at the $n$th symbol time reads

$$
\begin{aligned}
\mathbf{y}_{j}^{\mathrm{tr}}[n]= & \sqrt{P_{\mathrm{tr}}} \sum_{i=1}^{L} \mathbf{H}_{j i}[n] \boldsymbol{\Phi}[n]+\mathbf{z}_{j}[n] \\
= & \sqrt{P_{\mathrm{tr}}} \mathbf{H}_{j j}[n] \boldsymbol{\Phi}[n] \\
& +\sqrt{P_{\mathrm{tr}}} \sum_{i=1, i \neq j}^{L} \mathbf{H}_{j i}[n] \boldsymbol{\Phi}[n]+\mathbf{z}_{j}[n],
\end{aligned}
$$

where $P_{\text {tr }}$ is the training transmit power and " $\operatorname{tr}$ " denotes the training period. 

follows:

Then, the signal for channel estimation is derived as

$$
\begin{aligned}
\widetilde{\mathbf{y}}_{j k}^{\mathrm{tr}}[n]= & \frac{1}{\sqrt{P_{\mathrm{tr}}}} y_{j}^{\operatorname{tr}}[n] \Phi_{k}^{H}[n] \\
= & \mathbf{H}_{j j k}[n]+\sum_{i=1, i \neq j}^{L} \mathbf{H}_{j i k}[n] \\
& +\frac{1}{\sqrt{P_{\mathrm{tr}}}} \mathbf{z}_{j}[n] \Phi_{k}^{H}[n] .
\end{aligned}
$$

Here, $\mathbf{H}_{j j k}[n]$ is the desired channel, $\sum_{i=1, i \neq j}^{L} \mathbf{H}_{j i k}[n]$ is the so-called pilot contamination, and $\left(1 / \sqrt{P_{\mathrm{tr}}}\right) \mathbf{z}_{j}[n] \Phi_{k}^{H}$ denotes the effect of noise. For analysis, $\mathbf{R}_{j k}$ and $\Pi_{j k}$ are defined as $\mathbf{R}_{j k}=\sum_{i=1}^{L} \mathbf{R}_{j i k}$ and $\Pi_{j k}=\left(\delta_{b}^{2} / P_{\mathrm{tr}}\right) \mathbf{I}_{M}+\mathbf{R}_{j k}-\mathbf{R}_{j j k}$, respectively, where the subscript $j$ indicates the index of base station while the subscript $k$ indicates the index of UTs, and $\mathbf{z}_{j}[n] \Phi_{k}^{\mathbf{H}} \sim$ $\mathbb{C} \mathbb{N}\left(0, \delta_{b}^{2} \mathbf{I}_{M}\right)$.

So the real CSI can be described as

$$
\begin{aligned}
\mathbf{H}_{j j k}[n]= & \widetilde{\mathbf{y}}_{j k}^{\operatorname{tr}}[n] \\
& -\left(\sum_{i=1, i \neq j}^{L} \mathbf{H}_{j i k}[n]+\frac{1}{\sqrt{P_{\mathrm{tr}}}} \mathbf{z}_{j}[n] \boldsymbol{\Phi}_{k}^{H}\right) .
\end{aligned}
$$

By inserting (11) into (5), we derive

$$
\mathbf{H}_{j j k}[n+1]=\alpha \widetilde{\mathbf{y}}_{j k}^{\operatorname{tr}}[n]+\widetilde{\mathbf{e}}_{j j k}[n+1]
$$

where $\widetilde{\mathbf{e}}_{j j k}[n+1]=-\alpha\left(\sum_{i=1, i \neq j}^{L} \mathbf{H}_{j i k}[n]+\left(1 / \sqrt{P_{\text {tr }}}\right) \mathbf{z}_{j}[n] \Phi_{k}^{H}\right)+$ $\mathbf{e}_{j j k}[n+1]$.

$\widetilde{\mathbf{e}}_{j j \mathrm{k}}[n+1]$ consists of the interference from users in other cells, the Gaussian White noise, and channel aging $\mathbf{e}_{j j k}[n+$ 1]. In fact, the interference from users in other cells is the sum of the interference channel coefficients that are i.i.d. Gaussian variants with zero mean, and the channel aging error is assumed to be uncorrelated with the interference channel coefficients. This model for pilot contamination and noise is proposed and widely used in prior works $[8,12]$; here we extend it for training period in the presence of channel aging, pilot contamination, and noise. Therefore, $\widetilde{\mathbf{e}}_{j j k}[n+1]$ satisfies $\widetilde{\mathbf{e}}_{j j k}[n+1] \sim \mathbb{C N}\left(0, \mathbf{R}_{j j k}-\alpha^{2} \mathbf{R}_{j j k}+\alpha^{2} \Pi_{j k}\right)$.

Similar to Section 3.1, we can get

$$
\begin{aligned}
& \mathbf{y}_{j k}[n+1]-\mathbf{z}_{j}[n+1] \\
& \quad=\left(\alpha \sqrt{P_{u l}} \widetilde{\mathbf{y}}_{j k}^{\operatorname{tr}}[n]-\left(-\sqrt{P_{u l}} \widetilde{\mathbf{e}}_{j i k}[n+1]\right)\right) \mathbf{x}_{j k}[n+1],
\end{aligned}
$$

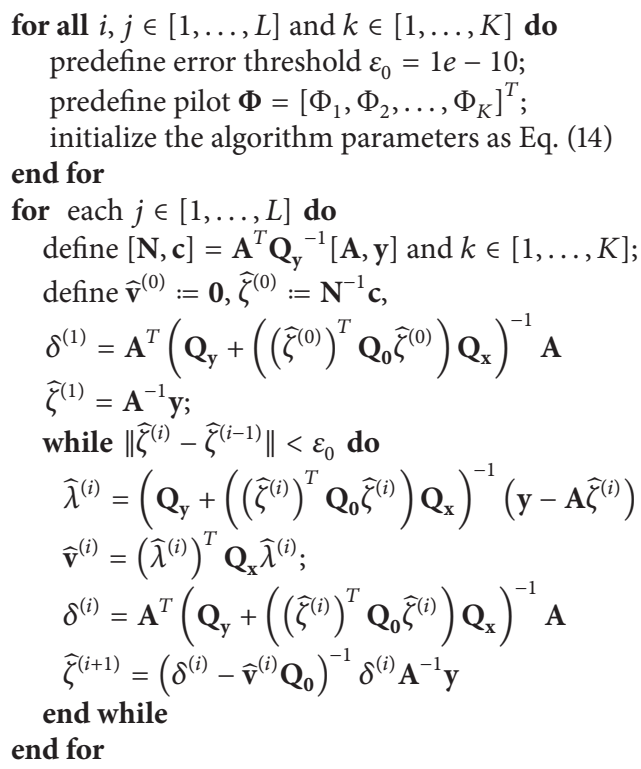

Algorithm 1: Signal Estimate Algorithm.

where we can also model it as an EIV model to estimate the transmitted signal more accurately. To get the estimated $\mathbf{x}_{j k}[n+1]$, we make the following definition:

$$
\begin{aligned}
\mathbf{y} & =\mathbf{y}_{j k}[n+1], \\
\mathbf{A} & =\alpha \sqrt{P_{u l}} \widetilde{\mathbf{y}}_{j k}^{\operatorname{tr}}[n], \\
\mathbf{e}_{y} & =\mathbf{z}_{j}[n+1], \\
\mathbf{E}_{\mathbf{A}} & =-\sqrt{P_{u l}} \widetilde{\mathbf{e}}_{j i k}[n+1], \\
\mathbf{Q}_{y} & =\delta_{0}^{2} \mathbf{I}_{M}, \\
\mathbf{Q}_{0} & =\mathbf{I}_{M}, \\
\mathbf{Q}_{x} & =P_{u l}\left(\mathbf{R}_{j j k}-\alpha^{2} \mathbf{R}_{j j k}+\alpha^{2} \Pi_{j k}\right) .
\end{aligned}
$$

In this model, we incorporate effect of pilot contamination with that of channel aging and use the proposed iterative Signal Estimate Algorithm to estimate the transmitted signal [9]. Comparing to the channel-aging-only scenario, the error of the channel model becomes larger. Apart from that, it is obvious that this model reduces to the pilot-contaminationonly scenario when $\alpha=1$. The procedures of the above iteration are outlined in Algorithm 1:

3.3. Channel Rectification. After the EIV model is established for pilot contamination and channel aging in Massive MIMO system in previous sections, the closed-form solution and the estimated parameters are complicated and hard to analytically compute, because the EIV model is essentially a nonlinear model. Here, we propose a channel rectification algorithm based on the weighted total least-squares solution 
(WTLSS) $[9,10]$, to solve this problem according to the theory of nonlinear least-squares adjustment.

While achieving more accurate signal estimation at the base station, the proposed algorithm can also rectify the channel model by deriving the residual error of channel; that is, $\widetilde{\mathbf{E}}_{\mathbf{A}}$, through the WTLSS method, can be expressed as (15).

$$
\begin{aligned}
\widetilde{\mathbf{E}}_{\mathbf{A}} & \\
= & -\mathbf{Q}_{x}\left(\mathbf{Q}_{y}+\left(\left(\widehat{\mathbf{x}}_{j k}[n+1]\right)^{T} \mathbf{Q}_{0} \widehat{\mathbf{x}}_{j k}[n+1]\right) \cdot \mathbf{Q}_{x}\right)^{-1} \\
& \cdot\left(\mathbf{y}-\mathbf{A} \widehat{\mathbf{x}}_{j k}[n+1]\right)\left(\widehat{\mathbf{x}}_{j k}[n+1]\right)^{T} \mathbf{Q}_{0} .
\end{aligned}
$$

Finally, the rectified channel model at the $(n+1)$ th symbol in Sections 3.1 and 3.2 is as follows:

$$
\mathbf{H}_{j j k}[n+1]=\alpha \boldsymbol{\omega}_{j j k}[n]-\frac{1}{\sqrt{P_{u l}}} \widetilde{\mathbf{E}}_{\mathbf{A}},
$$

where $\boldsymbol{\omega}_{j j k}[n]=\mathbf{H}_{j j k}[n]$ for Section 3.1; $\boldsymbol{\omega}_{j j k}[n]=\widetilde{\mathbf{y}}_{j k}^{\mathrm{tr}}[n]$ for Section 3.2. The contribution of this step is to update the channel estimated during the training period since the more accurate signal estimation corresponds to the more accurate channel model. Therefore, we can increase the time interval of transmitting pilot signals and decrease the expense of channel estimation.

\section{Simulation}

Here, simulations results are derived by Monte Carlo simulation technique, which averages over $10^{6}$ channel realizations $\left(10^{3}\right.$ different $R$ and each $R$ with $10^{3}$ different $v$ for $\left.h=R^{1 / 2} v\right)$. The effect of WTLSS method is compared with the traditional least square (LS) method. The estimation error $\|x-\widehat{x}\|^{2}$, where $x$ is the transmitted signal and $\widehat{x}$ is the estimation of the signal, is considered as the standard for comparison.

The following assumptions are made:

(1) $P_{\mathrm{tr}}=P_{u l}=P$.

(2) $\delta_{0}^{2}=1$ and the signal noise ratio SNR = $10 \log _{10}\left(\delta_{0}^{2} / P\right)$.

Figure 2 shows the effectiveness of WTLSS at different channel variations, in which the parameters is denoted by $f_{d} \times T_{s}$, when channel aging is the only factor taken into consideration. As pilot contamination and interference are assumed to be well removed here, the number of cell and the number of UT in each cell do not affect the accuracy of signal estimation. Figure 2 compares the signal estimation errors of the WTLSS method and the LS method when the number of antennas at the base station $\mathrm{Ar}=50,100$ and SNR $=10,30 \mathrm{~dB}$. It is obvious that WTLSS stands out compared to LS as $f_{d} \times T_{s}$ increases; that is, the channel changes substantially. When $f_{d} \times T_{s}$ is close to zero, WTLSS reduces to the traditional LS method. Increasing Ar and SNR does not improve its performance significantly but decreases the number of iterations of WTLSS method as is shown in Figure 3, which illustrates the number of WTLSS iterations versus $f_{d} \times T_{s}$.

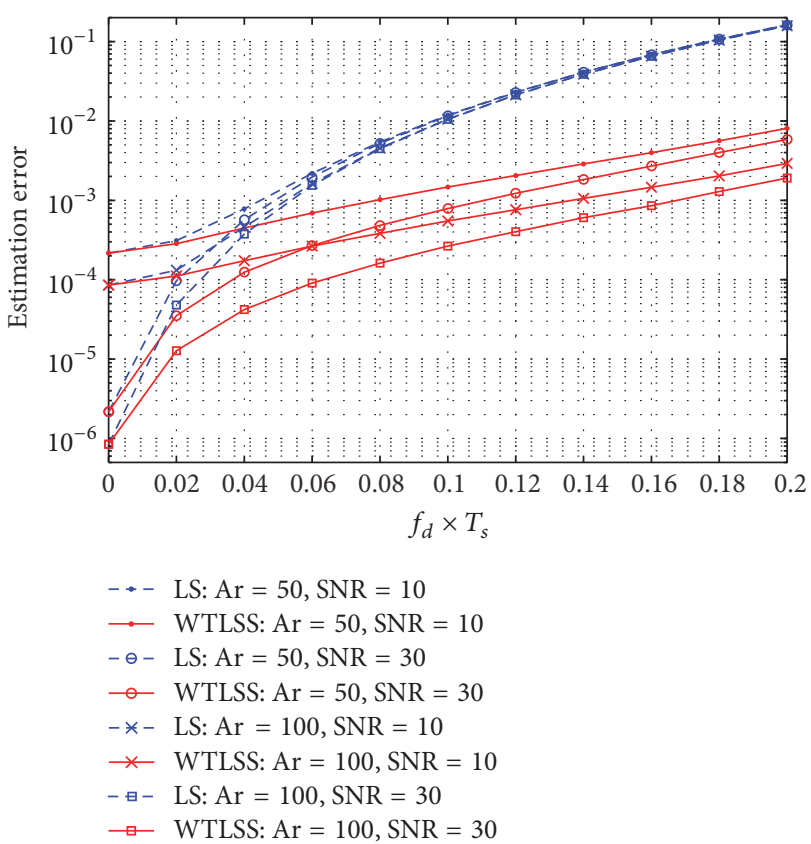

Figure 2: Signal estimation at different $f_{d} \times T_{s}$ with channel aging.

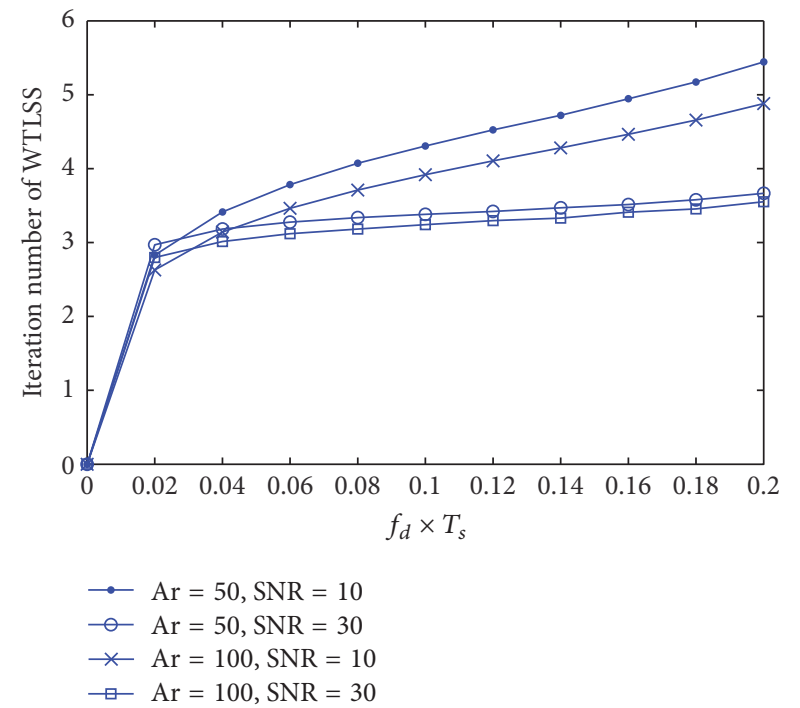

FIGURE 3: Iteration number for the convergence of WTLSS at different $f_{d} \times T_{s}$.

Figure 4 compares the signal estimation error of the WTLSS and LS methods when both channel aging and pilot contamination are considered in multicell Massive MIMO system. Here, we assume that $\mathrm{Ar}=50, \mathrm{SNR}=20 \mathrm{~dB}$, and $f_{d} \times T_{s}=0.2$. As is shown in the figure, the WTLSS method performs better than LS method. However, as the number of cells increases, the effect of pilot contamination becomes more severe.

Figure 5 presents the effects of WTLSS and LS methods taking both channel aging and pilot contamination into consideration with different transmission SNR of UTs. The distance between UT and its corresponding base station is 


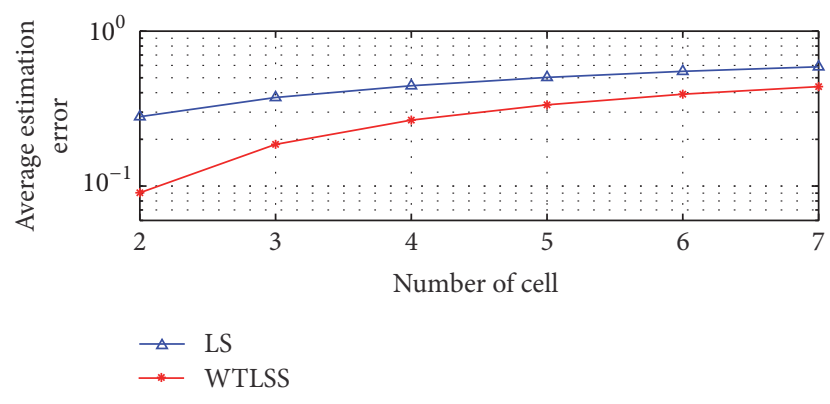

FIgURE 4: Signal estimation error of different number of cells with pilot contamination and channel aging.

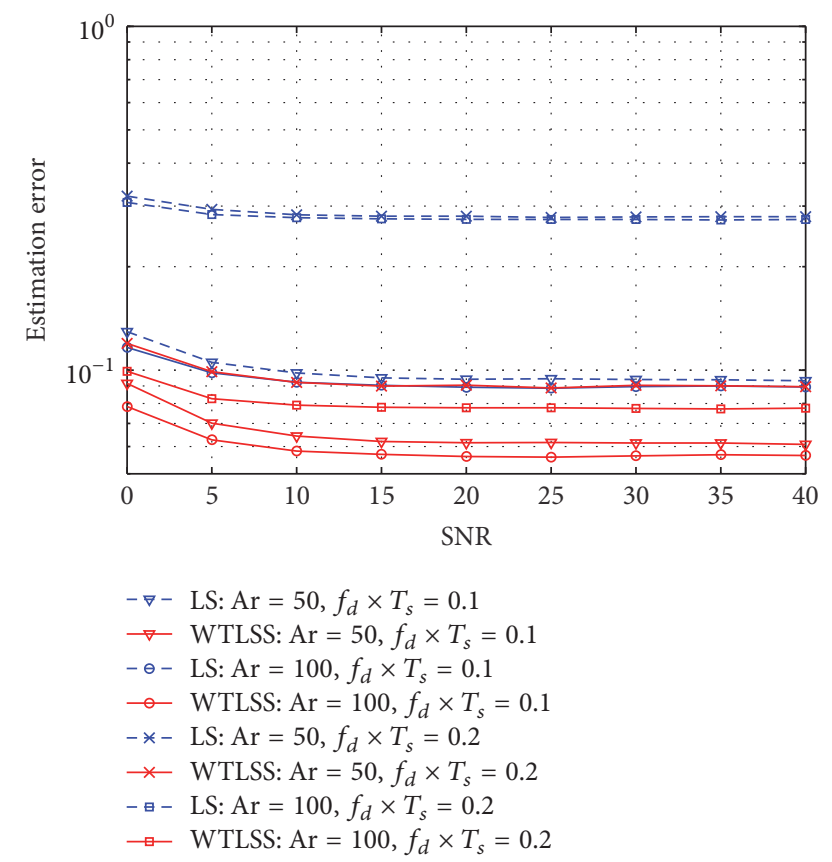

FIGURE 5: Signal estimation at different SNR with pilot contamination and channel aging.

assumed to be half less than the distance between UT and base stations in its adjacent cells; thus the power of aimed channel is four times larger than that of the "contamination" channel. As shown in Figure 5, WTLSS outperforms LS method for different transmission SNR in four different scenarios $\left(\mathrm{Ar} \in\{50,100\} ; f_{d} \times T_{s} \in\{0.1,0.2\}\right)$. As pilot contamination is not relevant to $f_{d} \times T_{s}$, the influence of the pilot contamination is increasing with the SNR increase; it is because the increased SNR in the adjacent cell will increase the "contamination" power. But when the value of SNR is large, the ratio of desired power and the "contamination" power will tend to be fixed, which will make the performance curve parallel to the $x$-axis. On the other side, the proposed algorithm decreases the reflection of the impact of channel aging. it is shown that the performance gap between different $f_{d} \times T_{s}$ using WTLSS is much less than the one using LS method.

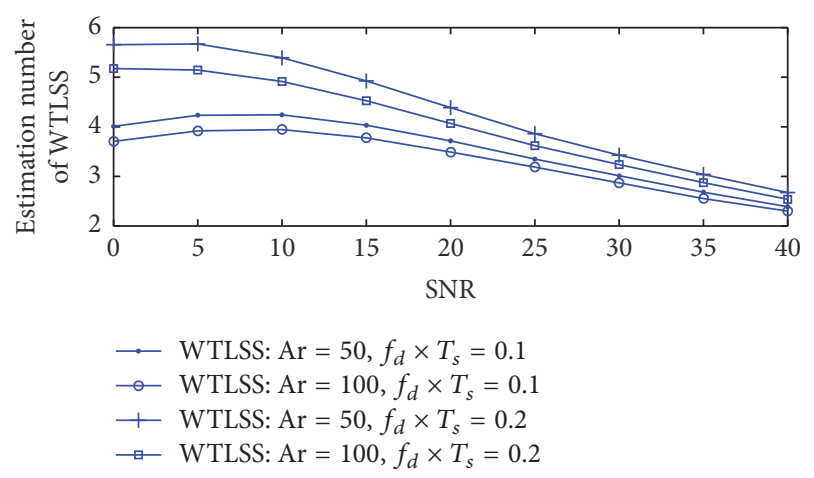

FIGURE 6: Signal estimation at different SNR with pilot contamination and channel aging.

Figure 6 shows that both increasing SNR and the smaller $f_{d} \times T_{s}$ will decrease the iteration number; it means that the complexity of the proposed iterative algorithm is influenced by the $f_{d} \times T_{s}$ and the SNR.

\section{Conclusion}

In this paper, we proposed an iterative method based on the WTLSS to alleviate the effect of channel aging and pilot contamination. We firstly incorporate these two effects and modeled them as an EIV model. Simulations are made to verify the efficiency of the proposed signal estimation algorithm compared with the traditional LS method. According to the simulation results, the proposed algorithm outperforms the LS method at both the channel-aging-only scenario and the scenario taking channel aging and pilot contamination into consideration, especially when $f_{d} \times T_{s}$ is large. The other advantage of our algorithm is that we can update the channel through deriving the channel estimate error, and the validity of channel rectification is also verified by simulation.

\section{Competing Interests}

The authors declare that there is no conflict of interests regarding the publication of this paper.

\section{Acknowledgments}

This work was supported by the National Natural Science Foundations of China under Grants 61601334 and 61201168 and by project of Natural Science Foundation of Hubei Province under Grant 2015CFC870.

\section{References}

[1] H. Q. Ngo, E. G. Larsson, and T. L. Marzetta, "Energy and spectral efficiency of very large multiuser MIMO systems," IEEE Transactions on Communications, vol. 61, no. 4, pp. 1436-1449, 2013.

[2] E. G. Larsson, O. Edfors, F. Tufvesson, and T. L. Marzetta, "Massive MIMO for next generation wireless systems," IEEE Communications Magazine, vol. 52, no. 2, pp. 186-195, 2014.

[3] H. Wang, Z. Pan, J. Ni, and I. Chih-Lin, "A spatial domain based method against pilot contamination for multi-cell massive 
MIMO systems," in Proceedings of the 8th International Conference on Communications and Networking in China (CHINACOM '13), pp. 218-222, IEEE, Guilin, China, August 2013.

[4] P. Xu, J. Wang, and J. Wang, "Effect of pilot contamination on channel estimation in massive MIMO systems," in Proceedings of the International Conference on Wireless Communications and Signal Processing (WCSP '13), pp. 1-6, Hangzhou, China, October 2013.

[5] K. T. Truong and R. W. Heath, "Effects of channel aging in massive MIMO systems," Journal of Communications and Networks, vol. 15, no. 4, pp. 338-351, 2013.

[6] A. Farhang, A. Aminjavaheri, N. Marchetti, L. E. Doyle, and B. Farhang-Boroujeny, "Pilot decontamination in CMT-based massive MIMO networks," in Proceedings of the 11th International Symposium on Wireless Communications Systems (ISWCS '14), pp. 589-593, IEEE, Barcelona, Spain, August 2014.

[7] M. A. Latif Sarker and M. H. Lee, "A fast channel estimation and the reduction of pilot contamination problem for massive MIMO based on a diagonal Jacket matrix," in Proceedings of the 4th International Workshop on Fiber Optics in Access Network (FOAN '13), pp. 26-30, Almaty, Kazakhstan, September 2013.

[8] M. Vu and A. Paulraj, "On the capacity of MIMO wireless channels with dynamic CSIT," IEEE Journal on Selected Areas in Communications, vol. 25, no. 7, pp. 1269-1283, 2007.

[9] B. Schaffrin and A. Wieser, "On weighted total least-squares adjustment for linear regression," Journal of Geodesy, vol. 82, no. 7, pp. 415-421, 2008.

[10] Y. Shen, B. Li, and Y. Chen, "An iterative solution of weighted total least-squares adjustment," Journal of Geodesy, vol. 85, no. 4, pp. 229-238, 2011.

[11] J. Hoydis, S. Ten Brink, and M. Debbah, "Massive MIMO in the UL/DL of cellular networks: how many antennas do we need?" IEEE Journal on Selected Areas in Communications, vol. 31, no. 2, pp. 160-171, 2013.

[12] H. Q. Ngo, T. L. Marzetta, and E. G. Larsson, "Analysis of the pilot contamination effect in very large multicell multiuser MIMO systems for physical channel models," in Proceedings of the IEEE International Conference on Acoustics, Speech and Signal Processing (ICASSP '11), pp. 3464-3467, IEEE, Prague, Czech Republic, May 2011. 


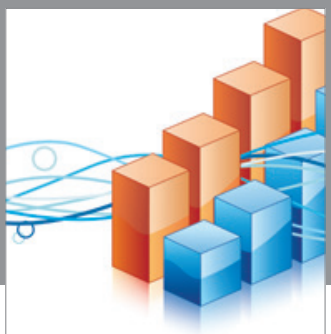

Advances in

Operations Research

vatem alat4

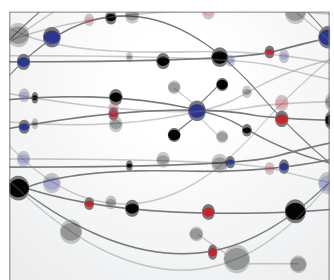

\section{The Scientific} World Journal
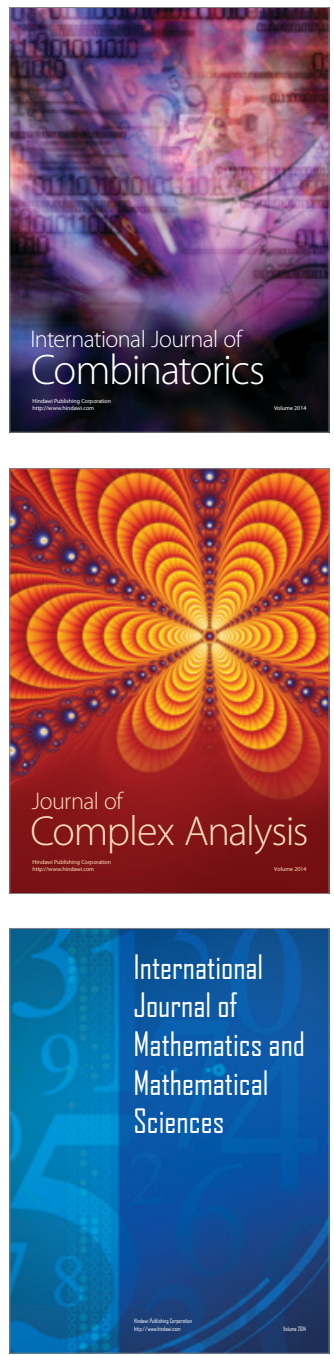
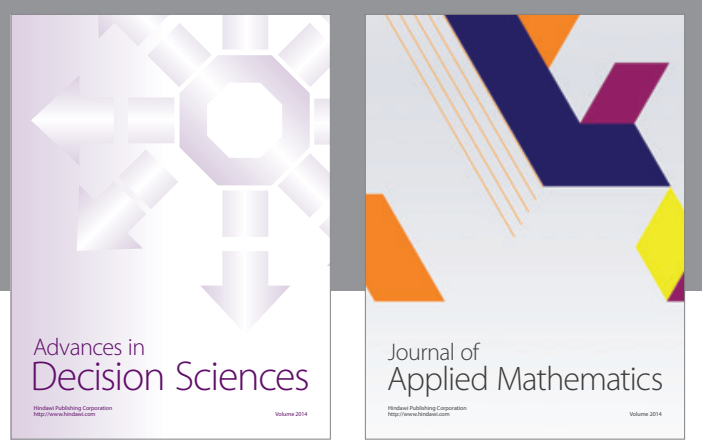

Algebra

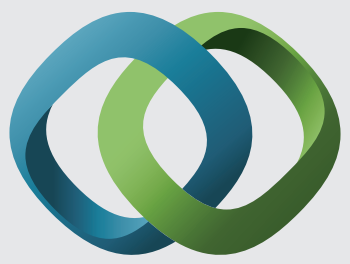

\section{Hindawi}

Submit your manuscripts at

https://www.hindawi.com
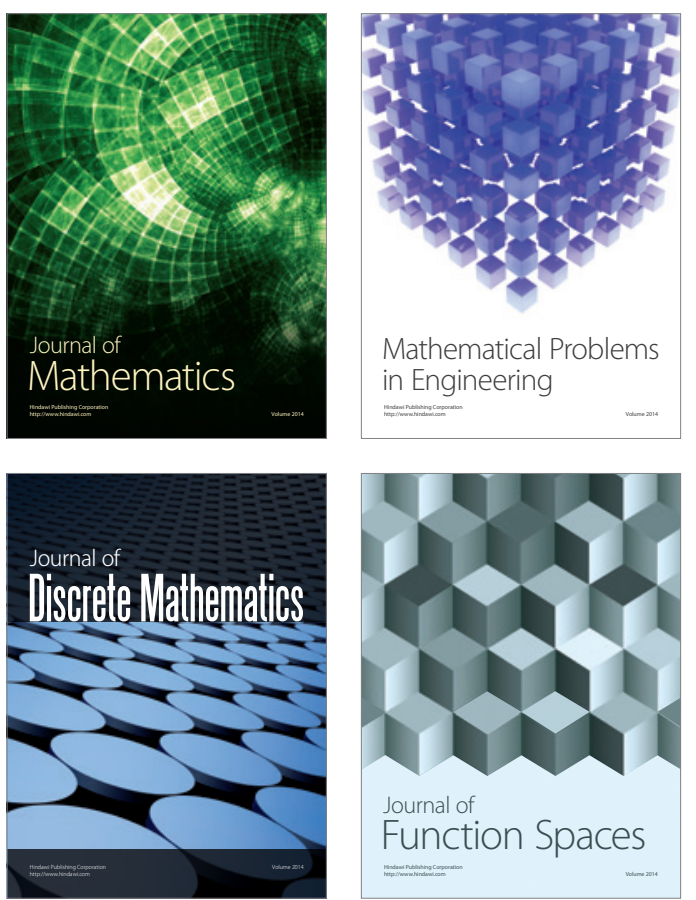

Mathematical Problems in Engineering
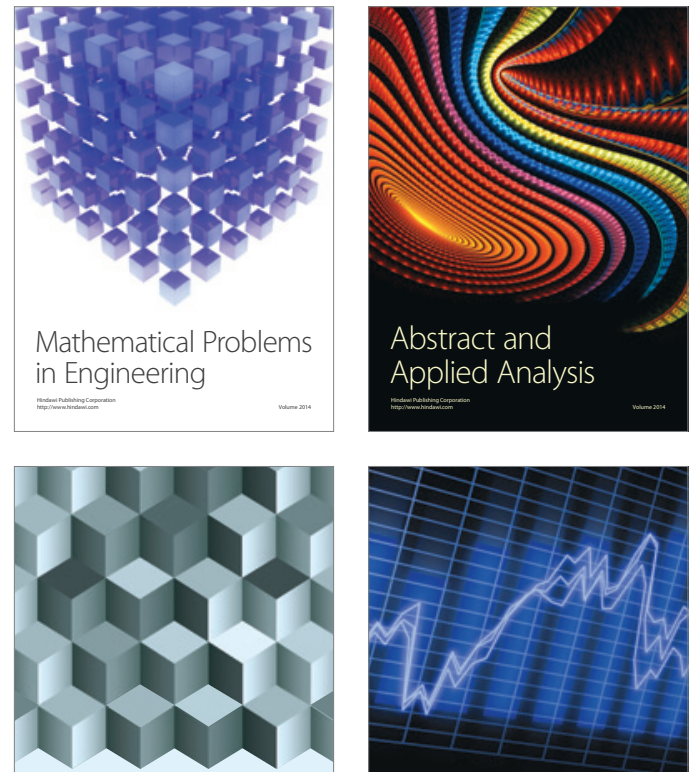

Journal of

Function Spaces

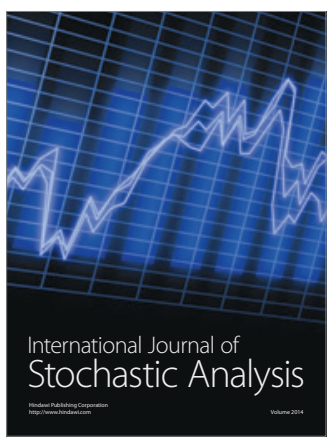

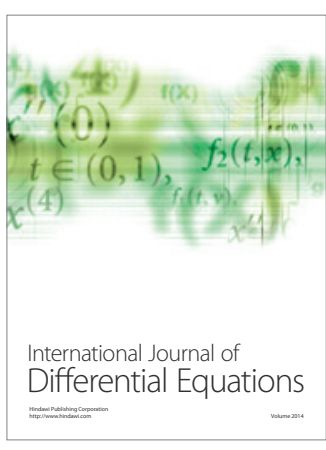
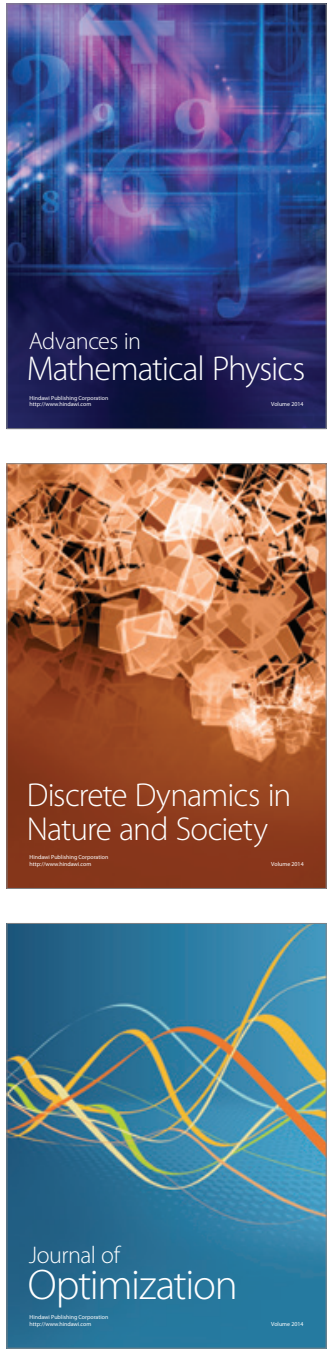